\title{
PROCEEDINGS
}

\section{A system with adjustable positive or negative thermal expansion}

\author{
By Joseph N. Grima*, Pierre S. Farrugia, Ruben Gatt \\ And Victor Zammit \\ Faculty of Science, University of Malta, Msida, MSD 06, Malta
}

\begin{abstract}
We analyse the anisotropic thermal expansion properties of a two-dimensional structurally rigid construct made from rods of different materials connected together through hinges to form triangular units. In particular, we show that this system may be made to exhibit negative thermal expansion coefficients along certain directions or thermal expansion coefficients that are even more positive than any of the component materials. The end product is a multifunctional system with tunable thermal properties that can be tailor-made for particular practical applications.
\end{abstract}

\section{Keywords: adjustable thermal expansion coefficient; negative thermal expansion;} thermal contraction

\section{Introduction}

The extent to which materials and structures deform when subjected to changes in temperature is a subject that has been studied for many years. Scientists and engineers must constantly account for temperature effects in their designs, as their neglect could result in various problems (Taylor 1998). Considerable advances are being made in the design, study and manufacture of materials and structures having very particular coefficients of thermal expansion, including materials and structures exhibiting negative coefficients of thermal expansion, i.e. materials which contract when heated (Lakes 1996; Mary et al. 1996; Sigmund \& Torquato 1996, 1997; Taylor 1998; Evans 1999; Milton 2002; Sleight 2002; Vandeperre et al. 2002; Vandeperre \& Clegg 2003; Barrera et al. 2005; Smith et al. 2005) and in recent years, various composites having predetermined coefficients of thermal expansion have been developed and are already in use in small-scale or high-tech applications that require a good match of the thermal properties (e.g. in teeth fillings (Versluis et al. 1996) or electronic applications (Holzer \& Dunand 1997)). Nevertheless, there is still the need to develop simpler and cheaper methods for achieving the same effect on any scale, particularly on a large scale.

Here, we discuss the properties of a simple structure (figure 1) which can be constructed at any length scale and exhibits the very interesting property that its thermal expansion coefficient can be controlled and adjusted to any pre-desired

* Author for correspondence (joseph.grima@um.edu.mt).

Electronic supplementary material is available at http://dx.doi.org/10.1098/rspa.2007.1841 or via http://www.journals.royalsoc.ac.uk. 


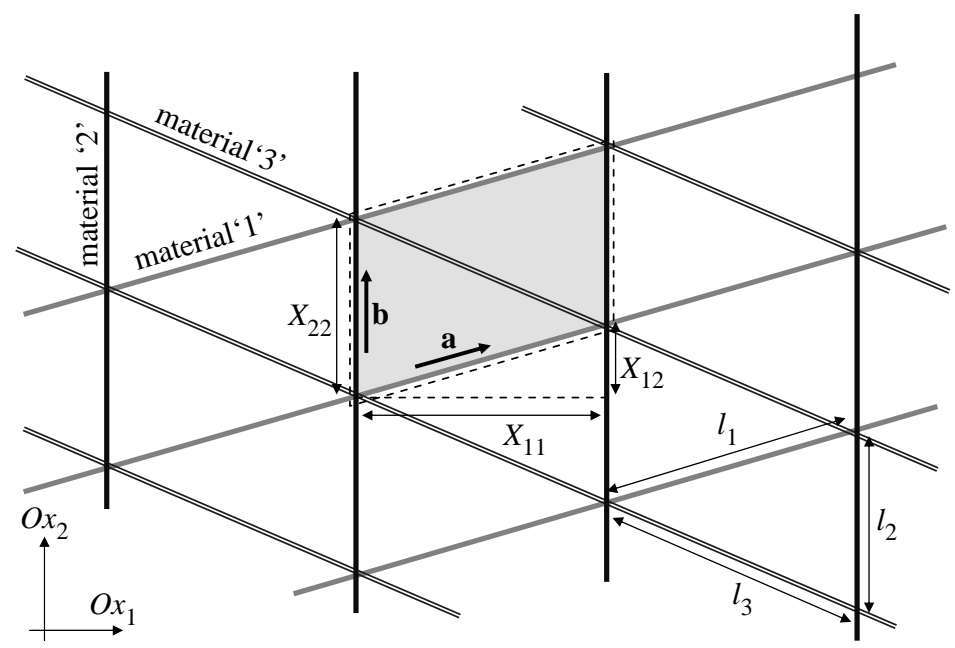

Figure 1. A two-dimensional lattice made of rods, intersecting at pin joints. The rods in each direction are made of a different material. The unit cell of the lattice is shown by the dashed parallelogram.

value. As illustrated in figure 1 , in its most general case, this structure can be described as a two-dimensional periodic network made from three sets of rods of different materials (materials $1-3$ ).

\section{Model for the thermal expansion properties at a temperature $T_{0}$}

We will assume that rods of the same materials are aligned parallel and equidistant from each other in such a way that the three sets of rods intersect through a 'pin joint' with each other to form triangles with side lengths $l_{1}, l_{2}$ and $l_{3}$ (where $l_{m}$ corresponds to the length of the side made from material $m$ ) as shown in figure 1. It has recently been shown that triangles having one side made from a different material than the other two sides can be made to exhibit negative thermal expansion (Vandeperre et al. 2002; Vandeperre \& Clegg 2003; Smith et al. 2005) and as we will show, the presence of these triangular units in our construct, potentially having all three sides with different lengths $l_{m}$ and made from different materials, is the key requirement for enabling full control of the thermal expansion properties.

The geometry of our construct may be described in terms of a parallelogrammic unit cell that contains two triangles. If the structure is aligned in space in such a way that the rods of material 2 are always parallel to the $O x_{2}$ direction, then the unit cell will have unit cell vectors $\mathbf{a}=\left(X_{11}, X_{12}\right)$ and $\mathbf{b}=\left(0, X_{22}\right)$, where $X_{11}, X_{22}$ and $X_{12}$ are given by

$$
\begin{gathered}
X_{11}=\frac{1}{2 l_{2}}\left[\left(l_{1}+l_{2}+l_{3}\right)\left(-l_{1}+l_{2}+l_{3}\right)\left(l_{1}-l_{2}+l_{3}\right)\left(l_{1}+l_{2}-l_{3}\right)\right]^{1 / 2}, \\
X_{22}=l_{2}, \\
X_{12}=\sqrt{l_{1}^{2}-X_{11}^{2}}=\frac{1}{2}\left(\frac{l_{1}^{2}-l_{3}^{2}}{l_{2}}+l_{2}\right) .
\end{gathered}
$$


Taking the most general case in which the materials have different coefficients of thermal expansion $\alpha_{\mathrm{S} m}$, when the structure is subjected to a change in temperature $\mathrm{d} T$, the lengths $l_{m}$ will vary by different amounts defined by d $l_{m}=l_{m} \alpha_{\mathrm{S} m} \mathrm{~d} T$. These changes in $l_{m}$ owing to a temperature variation will result in changes in size and shape of the macrostructure which may be quantified through the symmetric tensor $\alpha_{i j}(i, j=1,2)$ that describes the anisotropic thermal expansion of a twodimensional system (Nye 1957). The values of $\alpha_{i j}$ are defined as

$$
\varepsilon_{i j}=\alpha_{i j} \mathrm{~d} T,
$$

where $\varepsilon_{11}$ and $\varepsilon_{22}$ are the axial strains in the $O x_{1}$ and $O x_{2}$ directions, respectively, while $\varepsilon_{12}$ and $\varepsilon_{21}$ are equal to half the shear strain $\gamma$. These strains are given by

$$
\varepsilon_{11}=\frac{\mathrm{d} X_{11}}{X_{11}} ; \quad \varepsilon_{22}=\frac{\mathrm{d} X_{22}}{X_{22}} ; \quad \gamma=2 \varepsilon_{12}=2 \varepsilon_{21}=\frac{1}{X_{11}}\left[\mathrm{~d} X_{12}-\left(\frac{X_{12}}{X_{22}}\right) \mathrm{d} X_{22}\right] .
$$

Thus, since

$$
\mathrm{d} X_{i j}=\sum_{m=1}^{3} \frac{\partial X_{i j}}{\partial l_{m}} \mathrm{~d} l_{m}=\sum_{m=1}^{3} \frac{\partial X_{i j}}{\partial l_{m}} \alpha_{\mathrm{S} m} l_{m} \mathrm{~d} T \quad(i, j=1,2),
$$

from equations (2.1)-(2.6), the elements of the thermal expansion tensor $\alpha_{i j}$ can be simplified to

$$
\begin{gathered}
\alpha_{11}=\frac{\varepsilon_{11}}{\mathrm{~d} T}=\left[l_{1}^{2}\left(l_{2}^{2}+l_{3}^{2}\right) \alpha_{\mathrm{S} 1}+l_{2}^{2}\left(l_{1}^{2}+l_{3}^{2}\right) \alpha_{\mathrm{S} 2}+l_{3}^{2}\left(l_{1}^{2}+l_{2}^{2}\right) \alpha_{\mathrm{S} 3}\right] \frac{1}{2 l_{2}^{2} X_{11}^{2}} \\
-\left[2 l_{2}^{2} X_{11}^{2} \alpha_{\mathrm{S} 2}+l_{1}^{4} \alpha_{\mathrm{S} 1}+l_{2}^{4} \alpha_{\mathrm{S} 2}+l_{3}^{4} \alpha_{\mathrm{S} 3}\right] \frac{1}{2 l_{2}^{2} X_{11}^{2}}, \\
\alpha_{22}=\frac{\varepsilon_{22}}{\mathrm{~d} T}=\alpha_{\mathrm{S} 2},
\end{gathered}
$$

and

$$
\alpha_{12}=\alpha_{21}=\frac{\varepsilon_{12}}{\mathrm{~d} T}=\frac{1}{2} \frac{\gamma}{\mathrm{d} T}=\frac{l_{1}^{2}\left(\alpha_{\mathrm{S} 1}-\alpha_{\mathrm{S} 2}\right)-l_{3}^{2}\left(\alpha_{\mathrm{S} 3}-\alpha_{\mathrm{S} 2}\right)}{2 X_{11} l_{2}} .
$$

In addition, using standard axis transformation techniques (Nye 1957), we may also obtain $\alpha(\zeta)$, the coefficient of thermal expansion in a direction subtending an angle $\zeta$ to the $O x_{1}$,

$$
\alpha(\zeta)=\alpha_{11} \cos ^{2}(\zeta)+2 \alpha_{12} \sin (\zeta) \cos (\zeta)+\alpha_{22} \sin ^{2}(\zeta) .
$$

Furthermore, using the standard theory of principal strains (Gere 2001), we can identify the maximum and minimum thermal expansion coefficients afforded by the structure, which are given by

$$
\alpha(\zeta)_{\max / \min }=\frac{\alpha_{11}+\alpha_{22}}{2} \pm \sqrt{\left(\frac{\alpha_{11}-\alpha_{22}}{2}\right)^{2}+\alpha_{12}^{2}},
$$

that occur at mutually orthogonal directions which are oriented at an angle of $\zeta_{\max / \min }$ to the $O x_{i}$ axes where $\zeta_{\max / \min }$ is given by

$$
\zeta_{\max / \min }=\frac{1}{2} \tan ^{-1}\left(\frac{2 \alpha_{12}}{\alpha_{11}-\alpha_{22}}\right) .
$$




\section{Results and discussion}

Equations (2.7)-(2.10) suggest that, in general, the coefficient of thermal expansion $\alpha(\zeta)$ will depend on the following:

(i) the geometry of the system (i.e. the relative magnitudes of $l_{m}$ ),

(ii) the properties of the materials (i.e. the magnitudes of $\alpha_{\mathrm{S} m}$ ), and

(iii) the direction of measurement (i.e. the angle $\zeta$ ).

These equations also suggest that the change in shape and size of the macrostructure owing to a change in temperature may result in negative thermal expansion (i.e. thermal contraction) in certain directions. For example, assuming that the macrostructure is constructed using conventional materials having positive but different coefficients of thermal expansion, the structure will contract in the $O x_{1}$ direction when heated (i.e. $\alpha_{11}$ will assume negative values) if $2 l_{2}^{2} X_{11}^{2} \alpha_{\mathrm{S} 2}+$ $l_{1}^{4} \alpha_{\mathrm{S} 1}+l_{2}^{4} \alpha_{\mathrm{S} 2}+l_{3}^{4} \alpha_{\mathrm{S} 3}>l_{1}^{2}\left(l_{2}^{2}+l_{3}^{2}\right) \alpha_{\mathrm{S} 1}+l_{2}^{2}\left(l_{1}^{2}+l_{3}^{2}\right) \alpha_{\mathrm{S} 2}+l_{3}^{2}\left(l_{1}^{2}+l_{2}^{2}\right) \alpha_{\mathrm{S} 3}$. Equations (2.11) and (2.12) suggest the directions where the structure exhibits maximum/ minimum thermal expansion coefficients and that the signs/magnitudes of these coefficients depend on the magnitudes of $\alpha_{i j}$ (equations (2.7)-(2.9)), which in turn depend on the relative lengths and coefficients of thermal expansion of the rods. All these suggest that the thermal expansion properties may be completely controlled through the choice of the parameters $l_{m}$ and $\alpha_{\mathrm{S} m}$, and as a consequence, by careful choice of these parameters, one may engineer macrostructures that exhibit predetermined thermal expansion properties, thus enabling the construction of systems which are tailor-made for particular practical applications.

These equations also show that, in general, $\alpha_{12}$ is not zero and hence the macrostructure may shear when subjected to a change in temperature. The condition for the structure not to shear is $l_{1}^{2}\left(\alpha_{\mathrm{S} 1}-\alpha_{\mathrm{S} 2}\right)=l_{3}^{2}\left(\alpha_{\mathrm{S} 3}-\alpha_{\mathrm{S} 2}\right)$, and this can be satisfied by the trivial solution where all $\alpha_{\mathrm{S} m}$ have the same value, $\alpha_{\mathrm{S}}$. In fact, under such conditions, the macrostructure will be isotropic in-plane, with $\alpha(\zeta)=\alpha_{\mathrm{S}}$, and upon heating the macrostructure will expand while maintaining its aspect ratio in accordance with common expectation. Another system that will not shear is the special case when $\alpha_{\mathrm{S} 1}=\alpha_{\mathrm{S} 3}$ and the triangles are isosceles with $l_{1}=l_{3}$ as discussed further on.

In an attempt to understand more clearly the potential of this macrostructure as a system with variable thermal expansion and, in particular, as a system that can exhibit thermal contraction, we shall consider the following five special cases.

I. Equilateral triangles with $\alpha_{\mathrm{S} 1}=\alpha_{\mathrm{S} 3} \neq \alpha_{\mathrm{S} 2}$.

II. Equilateral triangles with $\alpha_{\mathrm{S} 1}=\alpha_{\mathrm{S} 2} \neq \alpha_{\mathrm{S} 3}$.

III. Equilateral triangles with $\alpha_{\mathrm{S} 1} \neq \alpha_{\mathrm{S} 2} \neq \alpha_{\mathrm{S} 3} \neq \alpha_{\mathrm{S} 1}$.

IV. Isosceles triangles with $l_{1}=l_{3} \neq l_{2}$ and $\alpha_{\mathrm{S} 1}=\alpha_{\mathrm{S} 3} \neq \alpha_{\mathrm{S} 2}$.

V. More general cases.

(a) Case I: special case when the triangles are equilateral and $\alpha_{S 1}=\alpha_{S 3} \neq \alpha_{S 2}$

Let us first consider the particular case where triangles are equilateral (having $\left.l_{m}=l\right)$ at the temperature of interest and the intrinsic coefficients of thermal expansion are such that $\alpha_{\mathrm{S} 1}=\alpha_{\mathrm{S} 3} \neq \alpha_{\mathrm{S} 2}$ (i.e. the rods aligned parallel to the $O x_{2}$ 

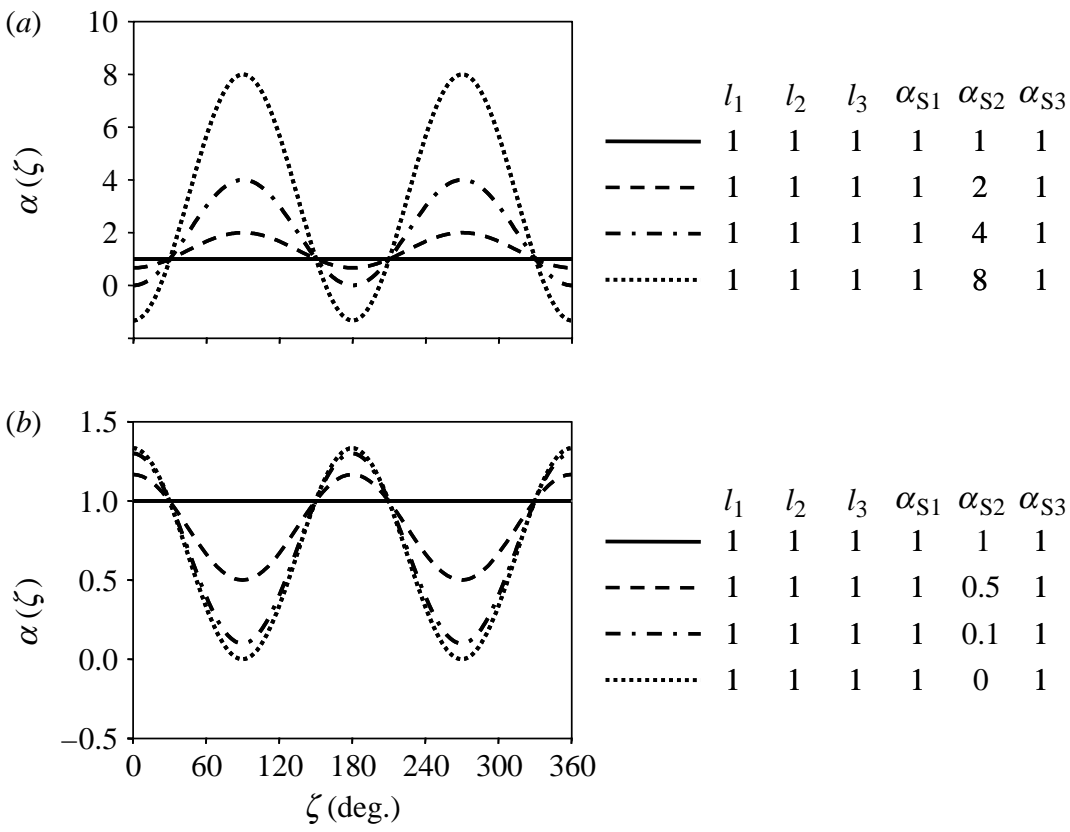

Figure 2. $(a, b)$ Typical plots of $\alpha(\zeta)$, the thermal expansion of the structure at an angle $\zeta$ to the $O x_{1}$ axes, against $\zeta$ for systems where the triangles are equilateral at the temperature of interest with one side having a different thermal expansion coefficient than the other two $\left(\alpha_{\mathrm{S} 1}=\alpha_{\mathrm{S} 3} \neq \alpha_{\mathrm{S} 2}\right.$, case I) .

direction have a thermal expansion coefficient that is different from the other two sets of rods).

In such a case, as illustrated in figure 2, the thermal expansion coefficients of the macrostructure will depend on the direction of measurement (i.e. it is anisotropic) and the particular values of $\alpha_{\mathrm{S} 1}$ and $\alpha_{\mathrm{S} 2}$. (As stated earlier, in the special case when $\alpha_{\mathrm{S} 1}=\alpha_{\mathrm{S} 2}=\alpha_{\mathrm{S} 3}$, the system will become isotropic where $\alpha(\zeta)=\alpha_{\mathrm{S} 1}=\alpha_{\mathrm{S} 2}=\alpha_{\mathrm{S} 3}$ as expected from a system made from a single isotropic material. In this respect, we note that isotropic behaviour can only be achieved when all rods are made from the same material.)

More specifically, when $l_{m}=l$ and $\alpha_{\mathrm{S} 1}=\alpha_{\mathrm{S} 3} \neq \alpha_{\mathrm{S} 2}$, equations (2.7)-(2.12) for the on-axis and off-axis values of the coefficient of thermal expansion for this system simplify to

$$
\begin{gathered}
\alpha_{11}=\frac{1}{3}\left(4 \alpha_{\mathrm{S} 1}-\alpha_{\mathrm{S} 2}\right), \\
\alpha_{22}=\alpha_{\mathrm{S} 2}, \\
\alpha_{12}=0, \\
\alpha(\zeta)=\alpha_{11} \cos ^{2}(\zeta)+\alpha_{22} \sin ^{2}(\zeta), \\
\alpha(\zeta)_{\max / \min }=\frac{2 \alpha_{\mathrm{S} 1}+\alpha_{\mathrm{S} 2}}{3} \pm \frac{2\left(\alpha_{\mathrm{S} 1}-\alpha_{\mathrm{S} 2}\right)}{3}=\frac{4 \alpha_{\mathrm{S} 1}-\alpha_{\mathrm{S} 2}}{3}, \quad \alpha_{\mathrm{S} 2}, \\
\zeta_{\max / \min }=\frac{1}{2} \tan ^{-1}\left(\frac{2 \alpha_{12}}{\alpha_{11}-\alpha_{22}}\right)=\frac{1}{2} \tan ^{-1}(0)=0^{\circ} .
\end{gathered}
$$


Equation (3.3) suggests that this system will not shear when heated, while equation (3.5) indicates that the maximum/minimum thermal expansion will always occur on-axis. Furthermore, from these equations, we can deduce the following.

(i) When $\alpha_{\mathrm{S} 1}=\alpha_{\mathrm{S} 3}<\alpha_{\mathrm{S} 2}$, minimum thermal expansion is exhibited in the $O x_{1}$ direction. This becomes negative when $4 \alpha_{\mathrm{S} 1}=4 \alpha_{\mathrm{S} 3}<\alpha_{\mathrm{S} 2}$. The magnitude of the negative thermal expansion can be increased by increasing $\alpha_{\mathrm{S} 2}$ relative to $\alpha_{\mathrm{S} 1}=$ $\alpha_{\mathrm{S} 3}$ (figure 2a). In addition, when $\alpha_{\mathrm{S} 1}=\alpha_{\mathrm{S} 3}<\alpha_{\mathrm{S} 2}$, the thermal expansion coefficient in the $O x_{2}$ direction $\alpha_{22}=\alpha_{\mathrm{S} 2}$ is the maximum (most positive) thermal expansion coefficient exhibited by the system.

(ii) When $\alpha_{\mathrm{S} 1}=\alpha_{\mathrm{S} 3}>\alpha_{\mathrm{S} 2}$, maximum thermal expansion is exhibited in the $O x_{1}$ direction where the maximum thermal expansion is greater than any of the individual $\alpha_{\mathrm{S} m} \mathrm{~S}$ (figure $2 b$ ). In addition, in this case, where $\alpha_{\mathrm{S} 1}=\alpha_{\mathrm{S} 3}>\alpha_{\mathrm{S} 2}$, the thermal expansion coefficient in the $O x_{2}$ direction $\alpha_{22}=\alpha_{\mathrm{S} 2}$ is the minimum (least positive) thermal expansion coefficient exhibited by the system.

All this is consistent with the work done by Smith et al. (2005). An animation illustrating this type of behaviour is supplied in the electronic supplementary material.

(b) Case II: special case when the triangles are equilateral and $\alpha_{S 1}=\alpha_{S 2} \neq \alpha_{S 3}$

It is important to note that the system in case I exhibits zero $\alpha_{12}$ and its maximum/minimum thermal expansion occurs on-axis due to the fact that the system is aligned in such a way that the rods which have a different thermal expansion from the other two sets (i.e. the rods of material 2) are set to be aligned parallel to the $O x_{2}$ direction (i.e. the $O x_{1}$ and $O x_{2}$ directions correspond to lines of symmetry). In fact, when this is not the case, for example, if the thermal expansion coefficients of the rods were such that $\alpha_{\mathrm{S} 1}=\alpha_{\mathrm{S} 2} \neq \alpha_{\mathrm{S} 3}$, then equations (2.7)-(2.12) simplify to

$$
\begin{gathered}
\alpha_{11}=\frac{1}{3}\left(\alpha_{\mathrm{S} 1}+2 \alpha_{\mathrm{S} 3}\right), \\
\alpha_{22}=\alpha_{\mathrm{S} 1} \\
\alpha_{12}=\frac{\alpha_{\mathrm{S} 1}-\alpha_{\mathrm{S} 3}}{\sqrt{3}}, \\
\alpha(\zeta)=\alpha_{11} \cos ^{2}(\zeta)+2 \alpha_{12} \sin (\zeta) \cos (\zeta)+\alpha_{22} \sin ^{2}(\zeta), \\
\alpha(\zeta)_{\max / \min }=\frac{2 \alpha_{\mathrm{S} 1}+\alpha_{\mathrm{S} 3} \pm \frac{2}{3}\left(\alpha_{\mathrm{S} 1}-\alpha_{\mathrm{S} 3}\right)=\frac{4 \alpha_{\mathrm{S} 1}-\alpha_{\mathrm{S} 3}}{3}, \quad \alpha_{\mathrm{S} 3},}{3} \zeta_{\max / \min }=\frac{1}{2} \tan ^{-1}\left(\frac{2 \alpha_{12}}{\alpha_{11}-\alpha_{22}}\right)=\frac{1}{2} \tan ^{-1}(-\sqrt{3})=-30^{\circ} .
\end{gathered}
$$

Equation (3.9) suggests that, in this case, the shear component is not zero and the extent of shear deformation when the structure experiences a temperature change is proportional to the differences between the thermal expansion 
coefficients of rods of materials 1 and 3 (assuming that the rods of material 2 remain aligned with the $O x_{2}$ direction). This can be attributed to the fact that the $O x_{1}$ direction does not correspond to a line of symmetry as was the case in case I. In addition, from equations (3.7) and (3.8) we may deduce that for this system, the on-axis thermal expansion coefficients will never be negative. However, negative thermal expansion is still possible and, as illustrated from equation (3.11), the directions of maximum/minimum thermal expansion coefficients are at $-30^{\circ}$ to the $O x_{1}$ and $O x_{2}$ axes, i.e. in directions that are normal and orthogonal to the rods which have a different thermal expansion from the other two sets (i.e. the rods made from material 3). In fact, if $\alpha_{\mathrm{S} 3}>$ $4 \alpha_{\mathrm{S} 1}=4 \alpha_{\mathrm{S} 2}$, then the system will exhibit negative thermal expansion which is at a maximum at $-30^{\circ}$ to the $O x_{2}$ direction, i.e. a direction which is orthogonal to the rod of material 3. Note that these conclusions can also be reached by considering that the system in case II is the equivalent of the system in case I after this is rotated by $60^{\circ}$. In fact, the plots of $\alpha(\zeta)$ for this system (not shown) will be equivalent to the ones in figure 2 with the difference that they would be $60^{\circ}$ out of phase. A similar discussion can be made for the system containing equilateral triangles where $\alpha_{\mathrm{S} 2}=\alpha_{\mathrm{S} 3} \neq \alpha_{\mathrm{S} 1}$.

(c) Case III: special case when the triangles are equilateral and $\alpha_{S 1} \neq \alpha_{S 2} \neq \alpha_{S 3} \neq \alpha_{S 1}$

If we were to consider a more general scenario where the triangles are still equilateral at the temperature of interest but the three sides have different thermal expansion coefficients (i.e. $\alpha_{\mathrm{S} 1} \neq \alpha_{\mathrm{S} 2} \neq \alpha_{\mathrm{S} 3} \neq \alpha_{\mathrm{S} 1}$ ), then equations $(2.7)-(2.12)$ will simplify to

$$
\begin{gathered}
\alpha_{11}=\frac{1}{3}\left(2 \alpha_{\mathrm{S} 1}-\alpha_{\mathrm{S} 2}+2 \alpha_{\mathrm{S} 3}\right), \\
\alpha_{22}=\alpha_{\mathrm{S} 2}, \\
\alpha_{12}=\frac{\alpha_{\mathrm{S} 1}-\alpha_{\mathrm{S} 3}}{\sqrt{3}}, \\
\alpha(\zeta)=\alpha_{11} \cos ^{2}(\zeta)+2 \alpha_{12} \sin (\zeta) \cos (\zeta)+\alpha_{22} \sin ^{2}(\zeta), \\
\alpha(\zeta)_{\max / \min }=\frac{\alpha_{\mathrm{S} 1}+\alpha_{\mathrm{S} 2}+\alpha_{\mathrm{S} 3}}{3} \pm \frac{1}{3} \sqrt{\left(\alpha_{\mathrm{S} 1}-2 \alpha_{\mathrm{S} 2}+\alpha_{\mathrm{S} 3}\right)^{2}+3\left(\alpha_{\mathrm{S} 1}-\alpha_{\mathrm{S} 3}\right)^{2}}, \\
\zeta_{\max / \min }=\frac{1}{2} \tan ^{-1}\left[\frac{\sqrt{3}\left(\alpha_{\mathrm{S} 1}-\alpha_{\mathrm{S} 3}\right)}{\alpha_{\mathrm{S} 1}+\alpha_{\mathrm{S} 3}-2 \alpha_{\mathrm{S} 2}}\right] .
\end{gathered}
$$

These equations suggest that as in case II, this structure will shear when it experiences a change in temperature where the extent of shear deformation is proportional to the differences between the thermal expansion coefficients of materials 1 and 3 (assuming that the system is still aligned in such a way that the rods of material 2 are always parallel to the $O x_{2}$ direction). This shearing can once again be attributed to the fact that the $O x_{1}$ direction does not correspond to a line of symmetry. 


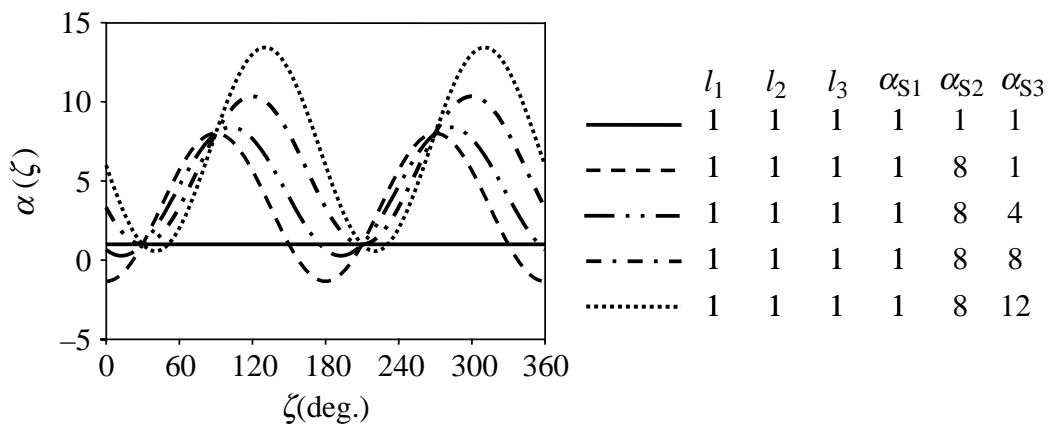

Figure 3. Typical plots of $\alpha(\zeta)$, the thermal expansion of the structure at an angle $\zeta$ to the $O x_{1}$ axes, against $\zeta$ for systems where the triangles are equilateral at the temperature of interest with all three sides having different thermal expansion coefficients from each other (case III).

In addition, we note that the system is still capable of exhibiting negative thermal expansion, even on-axis. For example, for negative thermal expansion in the $O x_{1}$ direction, we require that $\alpha_{\mathrm{S} 2}>2 \alpha_{\mathrm{S} 1}+2 \alpha_{\mathrm{S} 3}$. However, in this case, minimum/ maximum thermal expansion coefficients will not be exhibited on-axis and in fact the directions of maximum/minimum thermal expansion $\alpha(\zeta)$ will occur in the directions which are orthogonal to each other and at an angle of $\zeta_{\max / \min }$ to the $O x_{i}$ axis, where $\zeta_{\max / \min }$ is given by equation (3.17). Typical plots of $\alpha(\zeta)$ for various combinations of the intrinsic thermal expansion coefficients are shown in figure 3 . These plots highlight the increased anisotropy (when compared with case I, see figure 2) in the thermal expansion properties that results from using three materials having different thermal expansion coefficients rather than just two.

(d) Case IV: special case when the triangles are isosceles with $l_{1}=l_{3} \neq l_{2}$ and

$$
\alpha_{S 1}=\alpha_{S 3} \neq \alpha_{S 2}
$$

For a given set of rods with $\alpha_{\mathrm{S} 1}=\alpha_{\mathrm{S} 3} \neq \alpha_{\mathrm{S} 2}$, one can obtain a greater range of thermal expansion coefficients than those in case I by relaxing the condition that all the lengths $l_{m}$ are initially equal. To investigate this systematically, we will now consider the special case when the triangles are isosceles rather than equilateral with $l_{1}=l_{3} \neq l_{2}$ and $\alpha_{\mathrm{S} 1}=\alpha_{\mathrm{S} 3} \neq \alpha_{\mathrm{S} 2}$. In such cases, equations (2.7)-(2.12) will simplify to

$$
\begin{gathered}
\alpha_{11}=\frac{4 l_{1}^{2} \alpha_{\mathrm{S} 1}-l_{2}^{2} \alpha_{\mathrm{S} 2}}{4 X_{11}^{2}}=\frac{4 l_{1}^{2} \alpha_{\mathrm{S} 1}-l_{2}^{2} \alpha_{\mathrm{S} 2}}{4 l_{1}^{2}-l_{2}^{2}}, \\
\alpha_{22}=\alpha_{\mathrm{S} 2}, \\
\alpha_{12}=0, \\
\alpha(\zeta)=\alpha_{11} \cos ^{2}(\zeta)+\alpha_{22} \sin ^{2}(\zeta), \\
\alpha(\zeta)_{\max / \min }=\frac{4 l_{1}^{2} \alpha_{\mathrm{S} 1}-l_{2}^{2} \alpha_{\mathrm{S} 2}}{4 l_{1}^{2}-l_{2}^{2}}, \quad \alpha_{\mathrm{S} 2}, \\
\zeta_{\max / \min }=\frac{1}{2} \tan ^{-1}\left(\frac{2 \alpha_{12}}{\alpha_{11}-\alpha_{22}}\right)=\frac{1}{2} \tan ^{-1}(0)=0^{\circ} .
\end{gathered}
$$


From these equations, one may note that although this system shares various properties with the simpler system in case I where the triangles are equilateral (e.g. system does not shear and maximum/minimum thermal expansion occurs on-axis), in the current case, more extreme properties can be observed. In particular,

(i) when $\alpha_{\mathrm{S} 1}=\alpha_{\mathrm{S} 3}<\alpha_{\mathrm{S} 2}$, minimum thermal expansion is exhibited in the $O x_{1}$ direction (as was the case for equilateral triangles), where the minimum value of the thermal expansion may be lowered by (a) increasing the value of $\alpha_{\mathrm{S} 2}$ relative to $\alpha_{\mathrm{S} 1}=\alpha_{\mathrm{S} 3}$ (as was the case for the equilateral triangles), or (b) increasing $l_{2}$ relative to $l_{1}=l_{3}$. In fact, negative thermal expansion in the $O x_{1}$ direction can now be obtained when $\alpha_{\mathrm{S} 2}>4\left(l_{1}^{2} / l_{2}^{2}\right) \alpha_{\mathrm{S} 1}$ rather than $\alpha_{\mathrm{S} 2}>4 \alpha_{\mathrm{S} 1}$, as was the case for equilateral triangles. This is very significant, particularly from a manufacturing point of view, since as the ratio $l_{1} / l_{2}$ becomes smaller, it becomes possible for a structure to exhibit negative thermal expansion even if it is made from materials which have different though comparable values of $\alpha_{\mathrm{S} m}$. Furthermore, we note that very large negative thermal expansion coefficients can be exhibited in the limit when $l_{2} \rightarrow\left(l_{1}+l_{3}\right)$ and

(ii) when $\alpha_{\mathrm{S} 1}=\alpha_{\mathrm{S} 3}>\alpha_{\mathrm{S} 2}$, maximum thermal expansion is exhibited in the $O x_{1}$ direction (as was the case for equilateral triangles), and this maximum value may be increased by (a) decreasing the value of $\alpha_{\mathrm{S} 2}$ relative to $\alpha_{\mathrm{S} 1}=\alpha_{\mathrm{S} 3}$ (as was the case for the equilateral triangles), or (b) decreasing $l_{2}$ relative to $l_{1}=l_{3}$. Note that very large positive thermal expansion coefficients can be exhibited in the limit when $l_{2} \rightarrow\left(l_{1}+l_{3}\right)$, thus providing the useful property of thermal strain magnification, i.e. a system which exhibits very high strains for small changes in temperature. This can have very important applications, for example, as components of mechanical systems that respond to very small temperature changes.

Typical plots of $\alpha(\zeta)$ for various combinations of the intrinsic thermal expansion coefficients are shown in figure 4 . These plots highlight the wider range of possible values that the thermal expansion coefficients of this system can assume when compared with the system in case I (figure 2).

\section{(e) Case V: more general cases}

Although the special cases I-IV described above highlight the versatility of this structure, we note that equations (2.7)-(2.12), which apply to more general cases, suggest that, in reality, the manufacturer of such systems can control the properties of these systems through choice of any of the independent variables of the system (the relative lengths and the relative intrinsic thermal expansion coefficients of the materials used). In this respect, it is important to note that since $l_{m}$ can assume a continuous range of values, in practice, it would be easier to fine-tune the thermal expansion properties of the structure by altering the magnitudes of $l_{m}$ rather than by varying $\alpha_{\mathrm{S} m}$, since the range of values that $\alpha_{\mathrm{S} m}$ can assume are discreet and limited to those of available materials.

Typical plots of $\alpha(\zeta)$ for various combinations of lengths and the intrinsic thermal expansion coefficients are shown in figure 5. These clearly illustrate the versatility, anisotropicity and tunability of this connected rods system. An 
(a)
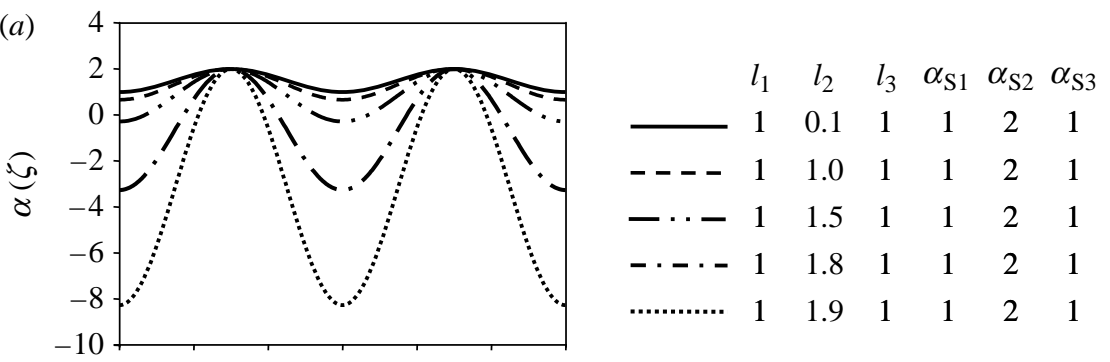

(b)
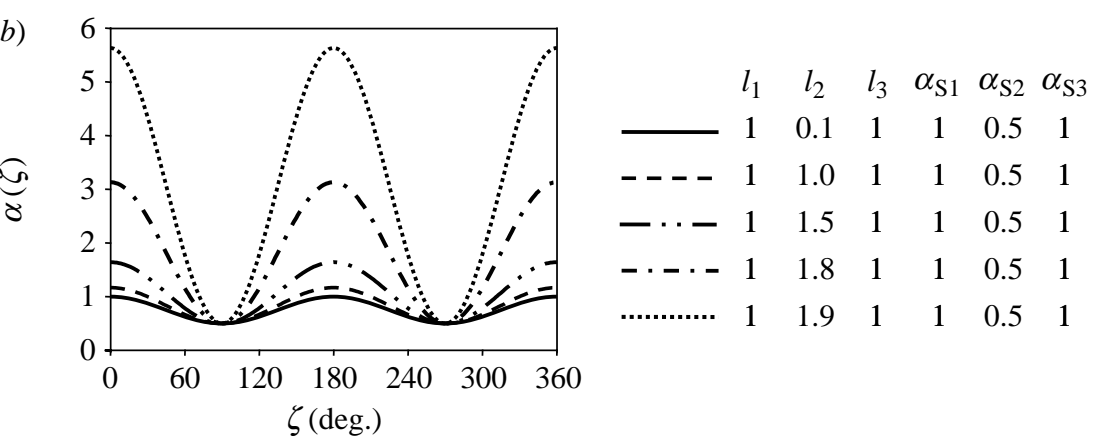

Figure 4. $(a, b)$ Typical plots of $\alpha(\zeta)$, the thermal expansion of the structure at an angle $\zeta$ to the $O x_{1}$ axes, against $\zeta$ for systems where the triangles are isosceles at the temperature of interest with the side of different length having a different thermal expansion coefficient than the other two $\left(l_{1}=l_{3} \neq l_{2}\right.$ and $\alpha_{\mathrm{S} 1}=\alpha_{\mathrm{S} 3} \neq \alpha_{\mathrm{S} 2}$, case IV $)$.

animation illustrating this more generalized behaviour is also supplied in the electronic supplementary material.

\section{The temperature dependence of the thermal expansion coefficients}

Referring to equations (2.7)-(2.12), it should be noted that since the values of the thermal expansion coefficients of the structure depend on the lengths $l_{m}$, in cases when at least one of the materials has a different $\alpha_{\mathrm{S} m}$ from the other two, the values of the thermal expansion coefficients of the structure will be dependent on the temperature since the relative magnitudes of $l_{m}$ are themselves dependent on the temperature. In fact, it is important to note that the thermal expansion properties discussed above are only valid for small temperature changes.

This temperature dependence of the coefficients of thermal expansion can have some very interesting consequences which, for example, may be illustrated by considering a special case of case I where at a temperature $T_{0}$, the triangles are equilateral with $\alpha_{\mathrm{S} 2}=4 \alpha_{\mathrm{S} 1}=4 \alpha_{\mathrm{S} 3}$. From equation (3.1), this system will exhibit zero thermal expansion $\alpha_{11}$ at $T=T_{0}$. However, as the temperature is increased, the lengths $l_{m}$ will increase in such a way that $l_{2}$ will always be longer than $l_{1}$ and $l_{3}$ for temperatures $T>T_{0}$, and conversely, if the temperature is decreased, the lengths $l_{m}$ will decrease in such a way that $l_{2}$ will always be shorter than $l_{1}$ and $l_{3}$ for temperatures $T<T_{0}$. In such cases, when $T \neq T_{0}$, the system will no longer be 

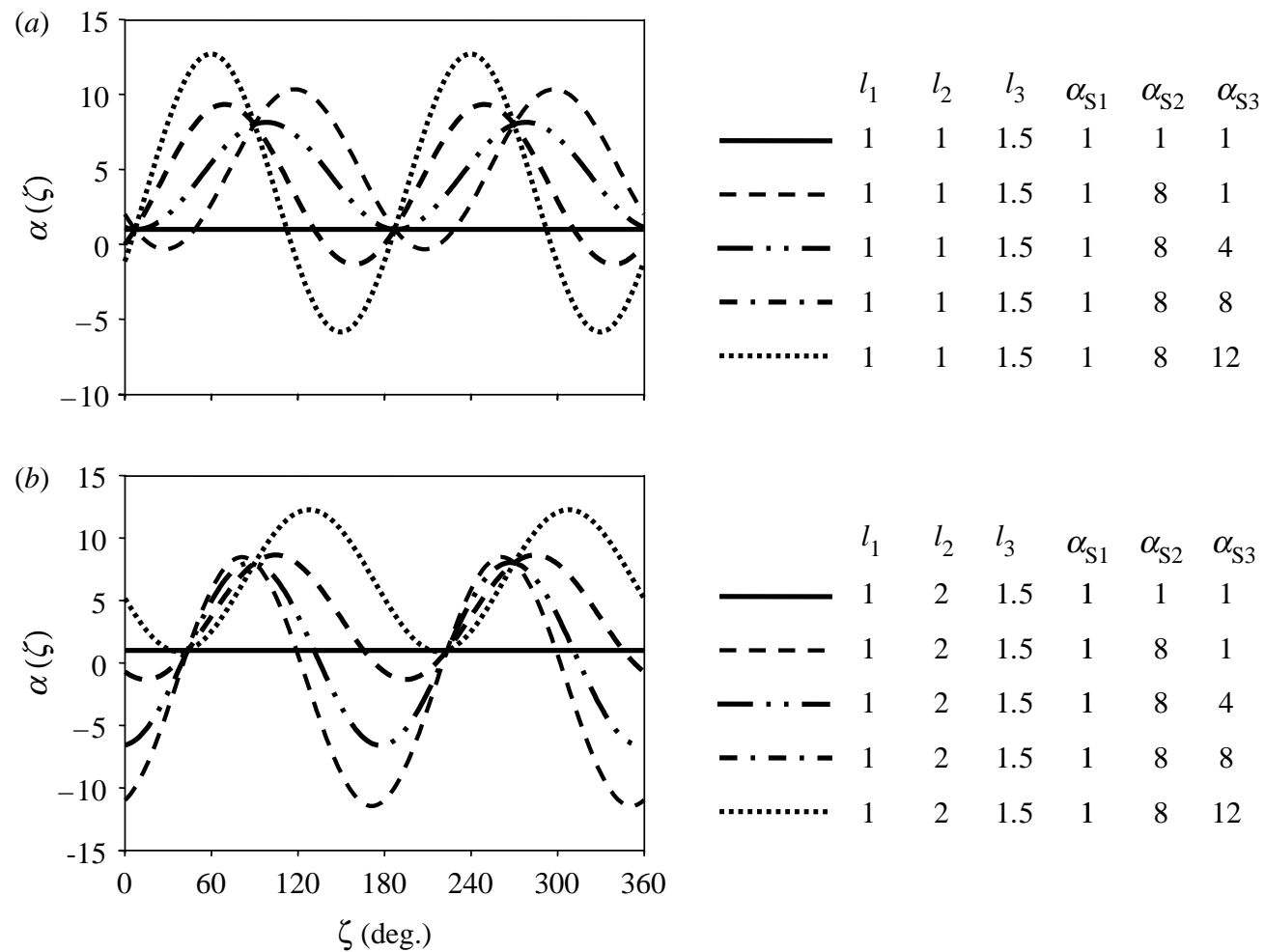

Figure 5. $(a, b)$ Typical plots of $\alpha(\zeta)$, the thermal expansion of the structure at an angle $\zeta$ to the $O x_{1}$ axes, against $\zeta$ for more general cases (case V).

represented by case I but by case IV, i.e. although the system exhibits zero thermal expansion $\alpha_{11}$ at $T=T_{0}$, it will exhibit negative $\alpha_{11}$ when $T>T_{0}$, which becomes more negative as $T$ is increased, and positive $\alpha_{11}$ when $T<T_{0}$.

\section{Conclusion}

In this study, we have modelled a system constructible at any scale, with adjustable thermal expansion. This system can exhibit very interesting and useful properties including thermal contraction (negative thermal expansion) and extreme thermal expansion properties, for example, a positive thermal expansion coefficient which is much more positive than any of the component materials. We have also shown that the thermal expansion properties of this system are highly anisotropic and temperature dependent. Given the simplicity of the construction (compared to other systems which can exhibit similar properties (Lakes 1996; Sigmund \& Torquato 1996, 1997; Milton 2002)), its adjustability and structural rigidity (since the construct under analysis consists of triangles which confer substantial structure rigidity), we envisage that the proposed construct or variations of it (including three-dimensional constructs where rods of different materials now form, for example, the edges of tetrahedra) should find extensive use in many practical applications. 
We acknowledge the financial support of the EU (through the FP6 CHISMACOMB project) and that of the Malta Council for Science and Technology (through its RTDI programme).

\section{References}

Barrera, G. D., Bruno, J. A. O., Barron, T. H. K. \& Allan, N. L. 2005 Negative thermal expansion. J. Phys.: Condens. Matter 17, R217-R252. (doi:10.1088/0953-8984/17/4/R03)

Evans, J. S. O. 1999 Negative thermal expansion materials. J. Chem. Soc. Dalton Trans. 19, 3317-3326. (doi:10.1039/a904297k)

Gere, J. M. 2001 Mechanical properties of materials, 5th edn. Cheltenham, UK: Nelson Thones Ltd.

Holzer, H. \& Dunand, D. 1997 Processing, structure and thermal expansion of metal matrix composites containing zirconium tungstate. In 4th Int. Conf. on Composite Engineering, Hawaii.

Lakes, R. S. 1996 Cellular solid structures with unbounded thermal expansion. J. Mater. Sci. Lett. 15, 475-477.

Mary, T., Evans, J. S. O., Vogt, T. \& Sleight, A. W. 1996 Negative thermal expansion from 0.3 to 1050 Kelvin in $\mathrm{ZrW}_{2} \mathrm{O}_{6}$. Science 272, 90-92. (doi:10.1126/science.272.5258.90)

Milton, G. 2002 The theory of composites. Cambridge, UK: Cambridge University Press.

Nye, J. F. 1957 Physical properties of crystals. Oxford, UK: Clarendon Press.

Sigmund, O. \& Torquato, S. 1996 Composites with extreme thermal expansion coefficients. Appl. Phys. Lett. 69, 3203-3205. (doi:10.1063/1.117961)

Sigmund, O. \& Torquato, S. 1997 Design of materials with extreme thermal expansion using a threephase topology optimization method. J. Mech. Phys. Solids 45, 1037-1067. (doi:10.1016/S00225096(96)00114-7)

Sleight, A. W. 2002 Negative thermal expansion. In Materials research society symposium proceedings, vol. 755 (eds M. Greenblatt, M. A. Alario-Franco, M. S. Whittingham \& G. Rohrer), p. DD10.6. Warrendale, PA: Materials Research Society.

Smith, C. W., Miller, W., Mackenzie, D. S. \& Evans, K. E. 2005 Mechanism for negative thermal expansion and its links to negative Poisson's ratio. Presented at 2nd Int. Workshop on A uxetic and Related Systems, Poznan, Poland.

Taylor, R. E. 1998 Thermal expansion of solids (ed. C. Y. Ho). ASM International.

Vandeperre, L. J. \& Clegg, W. J. 2003 Tailoring strains through microstructural design. In Materials research society symposium proceedings, vol. 785 (eds F. Furuya, E. Quandt, Q. Zhang, K. Inoue \& M. Shahinpoor), p. D114. Warrendale, PA: Materials Research Society.

Vandeperre, L. J., Howlett, A. \& Clegg, W. J. 2002 Application of negative thermal expansion to optical fibres. Presented at CIMTEC 2002: Int. Conf. on Modern Materials and Technologies, Florence, Italy.

Versluis, A., Douglas, W. H. \& Sakaguchi, R. L. 1996 Thermal expansion coefficient of dental composites measured with strain gauges. Dental Mater. 12, 290-294. (doi:10.1016/S0109$\underline{5641(96) 80036-4)}$ 